Published in final edited form as:

J Phys Chem B. 2019 May 02; 123(17): 3607-3617. doi:10.1021/acs.jpcb.9b00782.

\title{
Crowding-Induced Elongated Conformation of Urea-Unfolded Apoazurin: Investigating the Role of Crowder Shape in Silico
}

\author{
Fabio C. Zegarra ${ }^{\dagger, \uparrow}$, Dirar Homouz ${ }^{\dagger, \ddagger, \S, \uparrow, ~ A n d r e i ~ G . ~ G a s i c ~}{ }^{\dagger, \S}$, Lucas Babel $^{\dagger}$, Michael \\ Kovermann ${ }^{*}, \|, \perp$, Pernilla Wittung-Stafshede ${ }^{*}, \#$, Margaret S. Cheung ${ }^{*} \dagger, \S$ \\ tDepartment of Physics, University of Houston, Houston 77204, United States \\ ‡Department of Physics, Khalifa University of Science and Technology, Abu Dhabi, UAE \\ $\S$ Center for Theoretical Biological Physics, Rice University, Houston 77005, United States \\ "Department of Chemistry, Universität Konstanz, Konstanz 78464, Germany \\ ${ }^{\perp}$ Research School Chemical Biology (KoRS-CB), Universität Konstanz, Konstanz 78464, \\ Germany \\ \#Biology and Biological Engineering Department, Chalmers University, Göteborg 412 96, Sweden
}

\begin{abstract}
Here, we show by solution nuclear magnetic resonance measurements that the urea-unfolded protein apoazurin becomes elongated when the synthetic crowding agent dextran 20 is present, in contrast to the prediction from the macromolecular crowding effect based on the argument of volume exclusion. To explore the complex interactions beyond volume exclusion, we employed coarse-grained molecular dynamics simulations to explore the conformational ensemble of apoazurin in a box of monodisperse crowders under strong chemically denaturing conditions. The elongated conformation of unfolded apoazurin appears to result from the interplay of the effective attraction between the protein and crowders and the shape of the crowders. With a volumeconserving crowder model, we show that the crowder shape provides an anisotropic direction of the depletion force, in which a bundle of surrounding rodlike crowders stabilize an elongated conformation of unfolded apoazurin in the presence of effective attraction between the protein and crowders.
\end{abstract}

\section{Graphical abstract}

\footnotetext{
*Corresponding Authors michael.kovermann@uni-konstanz.de (M.K.)., pernilla.wittung@ chalmers.se (P.W.-S.)., mscheung@uh.edu (M.S.C.).

TF.C.Z. and D.H. contributed equally.

Author Contributions

M.K., P.W.-S., and M.S.C. designed research, F.C.Z., D.H., A.G.G., L.B., and M.K. performed research work and did data analysis. All authors contributed to discussions and data interpretation. M.S.C., P.W.-S., M.K. D.H., F.C.Z., and A.G.G. wrote the manuscript. ASSOCIATED CONTENT

Supporting Information

The Supporting Information is available free of charge on the ACS Publications website at DOI: 10.1021/acs.jpcb.9b00782. Hamiltonian of the coarse-grained protein model in the presence of urea, NMR measurements, definition of the asphericity $\Delta$, ratio of $I_{\mathrm{H}}$ and $R_{\mathrm{g}}$ as a function of $\Delta$, changes in the geometry of the protein as a function of volume fraction of crowders, and protein stability at different concentrations of urea (PDF)

The authors declare no competing financial interest.
} 


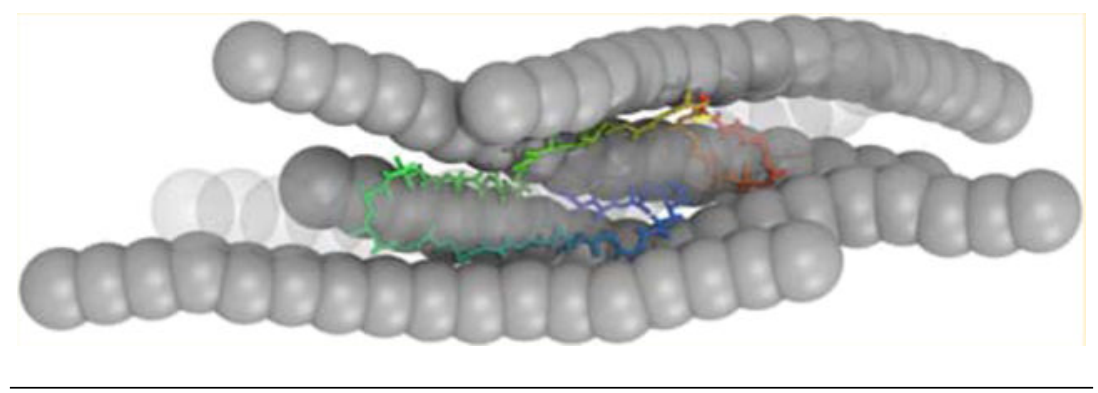

\section{INTRODUCTION}

The cellular environment is highly crowded with different types of macromolecules that can occupy volumes up to $40 \%$ of the total volume inside cells. ${ }^{1-3}$ To properly understand how proteins fold and function in vivo, it is necessary to consider the volume exclusion from surrounding macromolecules, known as the macromolecular crowding effect, ${ }^{4}$ which influences the structures and dynamics of a biopolymer in this restricted space. To test the crowding effect in vitro, sugar-based, branched polymers such as Ficoll and dextran ${ }^{5}$ are typically utilized as synthetic crowder agents to investigate the macromolecular crowding effect because they are considered to be chemically inactive agents in solution. Under the assumption that the dominant interaction between a crowding agent and a biopolymer is steric repulsion, the macromolecular crowding is shown to stabilize the conformation of compact biopolymers as shown in proteins, ${ }^{6-8}$ nucleic acids, ${ }^{9}$ and chromatins. ${ }^{10}$

However, here, we made the puzzling discovery by solution nuclear magnetic resonance (NMR) spectroscopy that our model protein, apoazurin, unfolded by a high urea concentration adopts more elongated conformations in the presence of dextran 20 than without a crowding agent (bulk). This is a deviation from a previous study from the WittungStafshede group where they have shown that apoazurin increases in stability with synthetic crowders. ${ }^{11}$ The thermodynamic stability of the two-state folding protein apoazurin increases with the increased volume fraction of crowders, and this effect was independent of the crowder size when various dextran sizes were analyzed, suggesting that a cylindrical representation from Minton's model ${ }^{12}$ is appropriate to model dextran that interacts with apoazurin through steric repulsions. ${ }^{13}$

To find an explanation to this counterintuitive result, we considered in silico coarse-grained molecular dynamics simulations, allowing more complex modeling of crowders than a simple hard sphere model for volume exclusion. The question central to all computational modeling is how to choose an appropriate level of the coarse graining of crowders that captures essential features without a prohibitive computational cost. All-atom molecular dynamics simulations have shown an unprecedented resolution of protein and protein crowders that allows investigation of subtle chemical interactions affecting protein stability and kinetics. ${ }^{14,15}$ These simulations demand massive computational resources and still require advanced sampling techniques to explore a wide range of phase space in a system for the computation of thermodynamic properties. ${ }^{16 ، 17}$ Coarse-grained models, although losing atomistic details, allow for greater sampling than in all-atom models and provide key 
polymeric insight into the distribution of protein conformations and the folding energy landscape under the macromolecular crowding effect. ${ }^{18-21}$

To investigate the structural ensemble of apoazurin in $6 \mathrm{M}$ urea in a periodic box of dextran crowders with coarse-grained molecular simulations, we must consider the appropriate dextran model. It is challenging to determine the geometry and property of dextran in a coarse-grained model because of its branched topology and polydisperse spatial distribution in solution. ${ }^{22}$ Early experiments based on filtration have characterized that the shapes of dextran crowders are asymmetrical by showing that dextrans pass through a pore much smaller than their presumed diameters. ${ }^{5}$ Sasahara et al. have further modeled the shape of dextrans as rods to fit the data from experimental measurement of the protein stability in dextrans with circular dichroism. ${ }^{12}$ Our previous work has adopted a spherocylindrical model for dextrans to explain the features of folding from various protein mutants. ${ }^{23}$ Nevertheless, these studies have assumed dextrans to interact with a protein through hardcore interactions. Interestingly, studies by Jiao et al. ${ }^{24}$ and, recently, by Ebbinghaus et al. ${ }^{25}$ showed that dextran stabilizes a protein through enthalpic interactions similar to its building block glucose, instead of stabilizing through volume exclusion. A suitable choice of dextran models again has become elusive from the past literature.

A mixture of dextran with destabilizing urea complicates our analysis of apoazurin folding. The net opposing impact from dextran and urea on protein stability can depend on the composition and become nonadditive. ${ }^{26}$ Given only the hydrodynamic radius of dextran in 6.8 M urea from the NMR experiments, our modeling approach was to vary the shape of a volume-conserving crowder model for dextran while changing the effective attraction between urea-unfolded apoazurin and its surrounding dextran crowders.

We first showed that a naive crowder model of hard-core repulsion between dextrans and apoazurin fails to capture the experimental observation. The addition of an effective attraction between dextrans and apoazurin is necessary to shift the protein ensemble toward elongated conformations. These effective attractions are unlikely the ones such as electrostatics and hydrogen bonding interactions found in protein crowders. ${ }^{27,28} \mathrm{We}$ are attracted to another possible explanation that compressible (physically soft) crowders ${ }^{29}$ or chemically soft repulsion ${ }^{30}$ produce effective attractions between a protein and a crowder because of the increased depletion between soft repulsive depletants. It is noteworthy that the soft repulsion from Zaccarelli's work ${ }^{29}$ is due to deformation of polymers, which is an entropic effect. In Harries's work, ${ }^{30,31}$ however, the soft repulsion from cosolute—solvent mixtures, resulting from changes in the hydrogen bonding in solvent near the first hydration shell, is represented by a step-like function in the interacting potential-an enthalpic effect. Our effective attractive potential can rise from either or both factors.

The geometry of an elongated rod model for dextrans further shifts the ensemble of apoazurin toward elongated conformations (favoring urea-unfolded conformations). The attraction between urea-unfolded apoazurin and dextrans facilitates dextrans to form aligned bundled conformations. The shape of the dextran breaks the symmetry of the depletion force, thus favoring an elongated geometry for the unfolded apoazurin. With coarse-grained 
modeling, we provide a plausible explanation to the puzzling discovery from the NMR experiments.

\section{MATERIALS AND METHODS}

\subsection{Apoazurin.}

We here used the apo-form Pseudomonas aeruginosa azurin, termed apoazurin, to study the excluded-volume effects of dextran 20 on the urea-denatured protein in vitro and in silico. It is a bacterial protein that binds a copper ion that can undergo oxidation-reduction as part of electron transfer chains in bacteria. Apoazurin has 128 residues, and the folded state adopts a $\beta$-sheet Greek-key fold with a disulfide bond (Cys3-Cys26) ${ }^{32}$ (see Figure 1). In vitro studies on apoazurin have advanced our knowledge on the folding mechanism of sandwichlike proteins. ${ }^{33}$ Apoazurin has provided an excellent template to compare experimental measurement with the predictions from protein folding theories ${ }^{34}$ and simulations. ${ }^{35}$

\subsection{NMR Measurements.}

All NMR experiments were conducted using a Bruker AVANCE III HD $850 \mathrm{MHz}$ spectrometer equipped with a $z$-gradient cryogenic probe using $20 \mathrm{mM} \mathrm{NaP}, \mathrm{pH} 7.0$ and $5 \%$ $\mathrm{D}_{2} \mathrm{O}(\mathrm{v} / \mathrm{v})$ as sample buffer. All one-dimensional data sets were processed and analyzed by TopSpin 3.2, whereas two-dimensional data sets were processed with NMRPipe ${ }^{38}$ and analyzed in NMRView. ${ }^{39}$ Water suppression was achieved by presaturation and WATERGATE.

2.2.1. Diffusion Experiments.-NMR diffusion spectra were recorded using pulsed field bipolar gradient stimulated echo experiments ${ }^{40}$ at $T=298 \mathrm{~K}$. For each diffusion profile, 21 different gradient strengths $G$ were used for $6 \mathrm{~ms}$ along the $z$ axis followed by a $100 \mathrm{~ms}$ recovery delay. The diffusion of apoazurin was allowed to proceed for $100 \mathrm{~ms}\left(c^{\mathrm{d} 20}\right.$ $=0 \mathrm{~g} / \mathrm{L}, c^{\mathrm{d} 20}=100 \mathrm{~g} / \mathrm{L}, c^{\mathrm{d} 20}=150 \mathrm{~g} / \mathrm{L}, c^{\mathrm{d} 20}=180 \mathrm{~g} / \mathrm{L}, c^{\mathrm{d} 20}=200 \mathrm{~g} / \mathrm{L}$ and $c^{\text {urea }}=0 \mathrm{M} ; c^{\mathrm{d} 20}$ $=0 \mathrm{~g} / \mathrm{L}, c^{\mathrm{d} 20}=100 \mathrm{~g} / \mathrm{L}$ and $\left.c^{\text {urea }}=6.8 \mathrm{M}\right), 150 \mathrm{~ms}\left(c^{\mathrm{d} 20}=150 \mathrm{~g} / \mathrm{L}\right.$ and $\left.c^{\text {urea }}=6.8 \mathrm{M}\right)$ or 200 ms $\left(c^{\mathrm{d} 20}=180 \mathrm{~g} / \mathrm{L}\right.$ and $\left.c^{\text {urea }}=6.8 \mathrm{M}\right)$. The calibration of $G$ was performed by a standard protocol. ${ }^{41}$ For error estimation, four different gradient strengths were repeated (relative gradient strengths of 1, 10, 40 and 70\%). The measured ${ }^{1} \mathrm{H}$ NMR spectra were integrated within the aliphatic signal region $I(0.5, \ldots, 1.5 \mathrm{ppm})$ and fitted to eq 1

$$
I(G)=I(0) \exp \left(-G^{2} \gamma^{2} \delta^{2} D(\Delta-\delta / 3)\right)
$$

where $\gamma$ is the gyromagnetic ratio, $\delta$ is the gradient length, $\Delta$ is the diffusion time, and $D$ is the calculated diffusion coefficient. ${ }^{40}$

2.2.2. Determination of Viscosity.-The viscosity $\eta$ of the solvent has been determined by measurement of the diffusion coefficient $D$ of the internal reference molecules dioxane present at different conditions $\left(c^{\mathrm{d} 20}=0 \mathrm{~g} / \mathrm{L}\right.$ and $c^{\text {urea }}=0 \mathrm{M} ; c^{\mathrm{d} 20}=0 \mathrm{~g} / \mathrm{L}$ and $\left.c^{\text {urea }}=6.8 \mathrm{M}\right)$ or dextran $20\left(c^{\mathrm{d} 20}=100 \mathrm{~g} / \mathrm{L}, c^{\mathrm{d} 20}=150 \mathrm{~g} / \mathrm{L}, c^{\mathrm{d} 20}=180 \mathrm{~g} / \mathrm{L}, c^{\mathrm{d} 20}=200\right.$ $\mathrm{g} / \mathrm{L}$ and $c^{\text {urea }}=0 \mathrm{M} ; c^{\mathrm{d} 20}=100 \mathrm{~g} / \mathrm{L}, c^{\mathrm{d} 20}=150 \mathrm{~g} / \mathrm{L}, c^{\mathrm{d} 20}=180 \mathrm{~g} / \mathrm{L}$ and $\left.c^{\text {urea }}=6.8 \mathrm{M}\right)$, 
known hydrodynamic radii of dioxane, $r_{\mathrm{H}}^{\text {dioxane }}=1.87 \AA^{42}$ and $r_{\mathrm{H}}^{\mathrm{d} 20}=32.4 \AA^{43}$ and by applying eq 2

$$
D=k_{\mathrm{B}} T /\left(6 \pi \eta r_{\mathrm{H}}\right)
$$

with Boltzmann's constant $k_{\mathrm{B}}$ and temperature $T$.

\subsection{Coarse-Grained Models.}

We have performed coarse-grained molecular simulations of apoazurin under several crowding conditions and under 0 and $6 \mathrm{M}$ of urea denaturant; $6 \mathrm{M}$ urea is a concentration that guarantees the denaturation of the protein at room temperature.

2.3.1. Coarse-Grained Apoazurin Model.-Our model protein apoazurin (PDB ID: 1E65; Figure 1) is studied employing a coarse-grained model. We coarse-grained each residue into a side chain and $\mathrm{C}_{a}$ bead with exception to glycine, which does not contain a side chain. ${ }^{44}$ The $\mathrm{C}_{a}$ bead is located at the position of the $a$-carbon, and the side-chain bead is located at the center of mass of the side-chain atoms. We have employed a structure-based Hamiltonian for our protein model, that is, the energy landscape of the protein follows the principle of minimal frustration, ${ }^{45-47}$ by following previous work. ${ }^{44,48}$ The dimensions of the protein are expressed in units of $\sigma(\sigma=3.8 \AA)$, which is the average distance between two consecutive $\mathrm{C}_{a}$ beads in a protein. This reduced unit of length is also used for the dimensions of the systems with crowders.

2.3.2. Coarse-Grained Dextran 20 Model.-We employed 12 volume-conserving models on dextran 20, a glucose-based polydisperse polymer of $20 \mathrm{kDa}$ molar mass, to investigate the effects of crowder shapes and effective attraction with a protein (Figure 2). The radius of $r_{\mathrm{H}}^{\mathrm{d} 20}=32.4 \AA$ was taken from the current experimental measurement. The rodlike shapes used by the models are spherocylinders ${ }^{21}$ with various ARs. A spherocylindrical model is created by connecting several overlapping spheres where the center-to-center distance between adjacent spheres is the radius of a sphere. The AR of a spherocylinder is defined as the ratio $L / D$, where $L$ is the distance between the centers of the two spheres located at the ends of the spherocylinder, and $D$ is the diameter of a sphere. For hard-core crowder models, the AR varies from 0 (DX20-S), to 1 (DX20-AR1), 2 (DX20AR2), 4 (DX20-AR4), 8 (DX20-AR8), and 16 (DX20-AR16), while keeping the volume the same as DX20-S with the radius of $r_{\mathrm{H}}^{\mathrm{d} 20}=32.4 \AA$ which is equivalent to $8.3 \sigma$. This value corresponds to the hydrodynamics radius of dextran 20 from our in vitro experiment, and it is approximately twice the radius of gyration $R_{\mathrm{g}}$ of the folded state $\left(R_{\mathrm{g}}^{\mathrm{N}} \approx 4 \sigma\right)$. The crowders with DX20-series interact with a protein through repulsion, and the crowders with DX20Aseries interact with the protein through attractive interactions. Table 1 shows the number of beads and radii of those beads needed to create the various ARs.

J Phys Chem B. Author manuscript; available in PMC 2019 November 21. 
An apoazurin protein is placed in the center of the box of crowders of the same type. The number of the crowders in a box depends on the volume fraction $\phi_{c}$ under study. The volume fractions studied are $\phi_{c}=0 \%$ (or bulk), 5,10 , and $20 \%$.

\subsubsection{Energy Function of Apoazurin under Urea Interference in a Periodic} Cubic Box of Crowders.-The Hamiltonian of the system $\left(E_{\mathrm{S}}\right)$ is the sum of the potential energy of the protein $\left(E_{\mathrm{p}}\right)$, the interaction between protein and crowders $\left(E_{\mathrm{pc}}\right)$, and the interaction between crowders $\left(E_{\mathrm{cc}}\right)$, that is, $E_{S}=E_{p}+E_{\mathrm{cc}}+E_{\mathrm{pc}} . E_{p}$ adopts a variant of structure-based potentials where only the contacts found in the native states are attractive according to the amino acid types, while the non-native contacts are repulsive. We have incorporated the chemical interference by adopting the "urea-SC-DIH" model from a previous study ${ }^{48}$ where the urea solvent-mediated interactions were included in the interactions between side-chain beads and the dihedral-potential that corresponds to four successive $C_{a}$ beads. The expression of $E_{\mathrm{p}}$ can be found in the Supporting Information.

For $E_{\mathrm{cc}}$, the interactions between crowders are repulsive and inversely related to the distance between the two crowders to the 12th power (eq S3). We set the interactions between protein and crowders, $E_{\mathrm{pc}}$, to be repulsive or attractive. The expression of repulsion between hard spheres of crowders labeled as DX20-series in Table 1 follows the same expression as $E_{\mathrm{cc}}$. The expression of nonspecific attraction between a crowder labeled as DX20A-series in Table 1 and a bead from the apoazurin model follows a Lennard-Jones potential with the depth well of $\varepsilon_{S}$. The relative strength of the attractive interaction is denoted by $\lambda=\varepsilon_{S} / \varepsilon$, where $\varepsilon=0.6 \mathrm{kcal} / \mathrm{mol}$ is the solvent-mediated interaction between Thr and Thr residues at $0 \mathrm{M}$. We used $\lambda=0.83$ and 2.50 for this study.

\subsection{Molecular Simulations.}

We used an in-house version of the Amber 10 molecular dynamics package ${ }^{49}$ to integrate the Langevin equation of motion in the low friction limit. ${ }^{50}$ The integration time step was set to $10^{-4} \tau_{\varepsilon}$, where $\tau_{\varepsilon}=\sqrt{\left(m \sigma^{2} / \varepsilon\right)}, m$ is the mass of a $\mathrm{C}_{a}$ bead, $\boldsymbol{\varepsilon}$ is the solvent-mediated interaction, and $\sigma$ is the van der Waals radius of a $\mathrm{C}_{a}$ bead. The replica exchange method $(\mathrm{REM})^{51,52}$ was used in order to efficiently sample the configurational space of samples when required. The exchanges between the neighboring replicas $i$ and $j$ are attempted every $40 \tau_{\varepsilon}$. The acceptance or rejection of each exchange follows the Metropolis criterion, $\left\{1, \exp \left[\left(\beta_{i}-\beta_{j}\right) \cdot\left(U\left(r_{i}\right)-U\left(r_{j}\right)\right)\right]\right\}$, where $\beta=1 / k_{\mathrm{B}} T, k_{\mathrm{B}}$ is the Boltzmann constant, $T$ is the temperature, and $U(r)$ is the potential energy of the system. Twenty replicas were employed where the temperature range used in a REM simulation varies for each condition. For each replica, more than 100000 statistically significant conformations were collected.

For each condition of the simulations with crowders, there are 10 sets of randomly assigned crowder configurations in a periodic boundary cubic box (PBC) as initial conditions. The size of PBC is $120 \times 120 \times 120 \sigma^{3}$ for the crowders with AR from 0 to 8 , and it is $185 \times 185$ $\times 185 \sigma^{3}$ for the crowders with $\mathrm{AR}=16$. The canonical simulations were performed at a temperature that corresponds to the in silico room temperature $T_{\mathrm{u}} . T_{\mathrm{u}}$ is obtained by transferring the difference between the melting temperature $\left(T_{\mathrm{m}}\right)$ and the room temperature 
( $298 \mathrm{~K}$ ) for an in vitro experiment to the in silico computational study as suggested in a prior study. ${ }^{35}$ The in silico room temperature is given by

$$
T_{\mathrm{u}}(\text { in silico })=T_{\mathrm{f}}(\text { in silico })-\left[T_{\mathrm{m}}(\text { in vitro })-298 \mathrm{~K}\right]
$$

where $T_{\mathrm{f}}$ is the in silico folding temperature. In our case, the in silico room temperature at 0 $\mathrm{M}$ urea is $k_{\mathrm{B}} T_{\mathrm{u}} / \varepsilon=1.14$.

\subsection{Data Analysis.}

2.5.1. Geometrical Properties of a Protein.-We measured the geometry of apoazurin by using the radius of gyration $\left(R_{\mathrm{g}}\right)$ and asphericity parameter $(\Delta)^{53}$ (see eq S7). $R_{\mathrm{g}}$ measures the size of the protein, and $\Delta$ measures the degree of anisotropy of the structure of the protein. $\Delta$ has the following range: $0 \leq \mathrm{A} \leq 1$, where a perfect sphere has $\Delta=0$. When $\Delta$ approaches 1 , it is an indication that the protein is fully extended.

2.5.2. Geometrical Properties of a Crowder Void.-The crowder void is defined as the space between the crowders in which the protein lies. To find this void, we partition the entire simulation box through Voronoi tessellation. ${ }^{54}$ From the tessellation, a 3D polygon is created around each bead (both crowders and protein residues). We select the crowder beads whose polygon is in contact with the polygon of the protein beads, and the positions of these selected crowder beads are used to define the vertices of the crowder void. We characterize the crowder void by computing two geometrical descriptors: the radius of gyration $R_{\mathrm{g}}$ and the asphericity parameter $\Delta$.

\section{RESULTS}

\subsection{Probing Apoazurin in Vitro.}

3.1.1. Structural Changes.-It has been established that high-resolution NMR spectroscopy enables the profound molecular characterization of the structural ${ }^{55}$ and dynamic properties ${ }^{56}$ of a protein ensemble as it is present in solution. We have made use of that by applying two-dimensional heteronuclear ${ }^{1} \mathrm{H}_{-}{ }^{15} \mathrm{~N}$ NMR experiments on apoazurin present under native and denatured conditions in a macromolecular crowding environment. Moreover, we have used pulse-field gradient (PFG)-NMR techniques to decode the potential impact of macromolecular crowding on the hydrodynamic dimension of apoazurin present under both folded and denatured conditions.

The addition of dextran 20 to apoazurin up to a concentration of $c^{\mathrm{d} 20}=200 \mathrm{~g} / \mathrm{L}$ changes neither the chemical shifts nor the signal intensities of apoazurin as probed by onedimensional proton (Figure S1) as well as by two-dimensional ${ }^{1} \mathrm{H}-{ }^{15} \mathrm{~N}$ NMR HSQC spectroscopy (Figure S2). This indicates that the addition of dextran 20 does not modify the structural properties of folded apoazurin at secondary and tertiary levels.

The maximum change in ${ }^{1} \mathrm{H},{ }^{15} \mathrm{~N}$ weighted chemical shift, $\Delta \omega_{\max }$, is $0.025 \mathrm{ppm}$ with an average $\Delta \omega^{\text {mean }}$ of $(0.012 \pm 0.004) \mathrm{ppm}$. Note that chemical shifts for aliphatic protons of apoazurin are highly conserved as well by comparing diluted and $c^{\mathrm{d} 20}=200 \mathrm{~g} / \mathrm{L}$ conditions 
for the folded state. Next, dextran 20 of up to $c^{\mathrm{d} 20}=180 \mathrm{~g} / \mathrm{L}$ was additionally supplemented to apoazurin that has been chemically unfolded using $c=6.8 \mathrm{M}$ of urea (Figures S3 and S4).

Again, changes in ${ }^{1} \mathrm{H},{ }^{15} \mathrm{~N}$ weighted chemical shift are small, with $\Delta \omega_{\max }=0.025 \mathrm{ppm}$ and $\Delta \omega^{\text {mean }}=(0.009 \pm 0.006) \mathrm{ppm}$. Chemical shifts for aliphatic protons are conserved in the presence of $c^{\text {urea }}=6.8 \mathrm{M}$ by comparing diluted and $c^{\mathrm{d} 20}=180 \mathrm{~g} / \mathrm{L}$ conditions as observed for folded apoazurin.

Taken together, $200 \mathrm{~g} / \mathrm{L}$ of dextran 20 does not change the structural integrity of the folded state and $180 \mathrm{~g} / \mathrm{L}$ of dextran 20 does not perturb the chemical shift pattern seen for chemically unfolded apoazurin. Consequently, we conclude that there is no significant solute-protein interaction between dextran 20 and apoazurin.

3.1.2. Molecular Dimension.-NMR diffusion methodology has been successfully employed for the profound characterization of the molecular dimensions of both folded and highly denatured proteins in solution. ${ }^{42}$ The initial idea with these experiments was to probe the expected (based on excluded volume theory) compaction of unfolded apoazurin when macromolecular crowding agents have been added.

First, we probed the diffusion properties of apoazurin in the absence of urea. The diffusion coefficient, $D$, obtained for folded apoazurin ranges from $D=(11.4 \pm 0.3) \times 10^{-11} \mathrm{~m}^{2} \mathrm{~s}^{-1}$ for diluted conditions to $D=(2.44 \pm 0.07) \times 10^{-11} \mathrm{~m}^{2} \mathrm{~s}^{-1}$ present at $c^{\mathrm{d} 20}=200 \mathrm{~g} / \mathrm{L}$ (Figure 2).

As the solvent viscosity $\eta$ increases from $\eta=0.89 \pm 0.01 \mathrm{mPa}$ s to $\eta=3.99 \pm 0.06 \mathrm{mPa} \mathrm{s}$ (Figure 3), the hydrodynamic radius $r_{\mathrm{H}}$ for folded apoazurin remains constant at $r_{\mathrm{H}}=21.7$ $\pm 0.8 \AA$. Notably, $r_{\mathrm{H}}$ determined for folded apoazurin is in excellent agreement at all concentrations of dextran 20 with the molecular dimension expected for a globular protein of azurin's size,${ }^{42}$ that is, $r_{\mathrm{H}}\left(N^{\mathrm{aa}}=128\right)=(20 \pm 5 \AA)($ Figure 4$)$.

Next, we probed the diffusion coefficient for apoazurin under denaturing conditions of $c^{\text {urea }}$ $=6.8 \mathrm{M}$. Again, $D$ decreases with an increasing concentration of dextran 20 from $D=(5.22$ $\pm 0.08) \times 10^{-11} \mathrm{~m}^{2} \mathrm{~s}^{-1}\left(c^{\mathrm{d} 20}=0 \mathrm{~g} / \mathrm{L}\right)$ to $D=(0.62 \pm 0.06) \times 10^{-11} \mathrm{~m}^{2} \mathrm{~s}^{-1}\left(c^{\mathrm{d} 20}=180 \mathrm{~g} / \mathrm{L}\right)$. However, the decrease in $D$ was not compensated by the increase in $\eta$ as found under native conditions. Instead, surprisingly, the hydrodynamic radius for denatured apoazurin increases from $r_{\mathrm{H}}=35.8 \pm 0.6 \AA\left(c^{\mathrm{d} 20}=0 \mathrm{~g} / \mathrm{L}\right)$ to $r_{\mathrm{H}}=46.6 \pm 2.5 \AA\left(c^{\mathrm{d} 20}=180 \mathrm{~g} / \mathrm{L}\right)($ Figure 4$)$.

Notably, $r_{\mathrm{H}}$, as determined here for denatured apoazurin under dilute conditions, agrees with the molecular dimensions expected for a denatured polypeptide chain of apoazurin's size ${ }^{42}$ $r_{H}\left(N^{\text {aa }}=128\right)=(35 \pm 18 \AA)$. Because excluded volume theory predicts that unfolded proteins become compacted in the presence of macromolecular crowding, more factors must be at play. To find a molecular-mechanistic explanation to this in vitro observation, we turned to in silico computations.

\subsection{In Silico Analysis of Apoazurin Unfolding.}

To compare our simulation results to the experimental measurements of apoazurin's hydrodynamic radii $r_{\mathrm{H}}$ under different conditions, we used two order parameters: radius of 
gyration $R_{\mathrm{g}}$ and asphericity $\Delta$. $R_{\mathrm{g}}$ is not an exact comparison to $I_{\mathrm{H}}$ because the shape of the object also matters in determining $I_{\mathrm{H}}$. For a perfect sphere, $I_{\mathrm{H}}$ can be approximated by $R_{g}$; however, as the protein becomes less spherical (or an increase in $\Delta$ ), $I_{\mathrm{H}}$ and $R_{\mathrm{g}}$ diverge (see Figure S5). $r_{\mathrm{H}} / R_{\mathrm{g}}$ can exceed 3 when $\Delta$ approaches 1 (a rod-like shape). This is due to the diffusion of spherical molecules being different from that of other elongated molecules. Therefore, $r_{\mathrm{H}}$ is in fact measuring a combination of the size and shape of a molecule.

\subsubsection{Structural Changes for the Repulsive Crowder Model and Chemical}

Denaturation.-We have investigated the ensemble conformations of apoazurin in various crowding contents at a chemically denatured state at $6 \mathrm{M}$ urea and at an in silico room temperature of $k_{\mathrm{B}} T_{\mathrm{u}} / \varepsilon=1.14$ (see Materials and Methods) with coarse-grained molecular simulations.

First, we have assumed that crowders and apoazurin interact through a repulsive potential that accounts for the excluded volume effects at $\phi_{\mathrm{c}}=5,10$, and $20 \%$. While conserving the volume of the crowders, we tested the shape effect by varying the AR of the crowders on apoazurin unfolding. Figure 5 a shows the average asphericity $(\Delta)$ normalized by the asphericity $\left(\Delta_{\mathrm{B}}\right)$ under the bulk condition for DX20-S, DX20-AR4, and DX20-AR8 against $\phi_{\mathrm{c}} . \Delta \Delta_{\mathrm{B}}$ are all less than 1 over a wide range of implying that all dextran 20 models with repulsive interactions with apoazurin do not contribute to the elongation of apoazurin. For most of the conditions, the macromolecular crowding effect leads to compaction of apoazurin with $\mathrm{R}_{\mathrm{g}} / \mathrm{R}_{\mathrm{g}, \mathrm{B}}$ less than 1 (Figure $5 \mathrm{~b}$ ). The crowders with a rodlike geometry show greater compaction than the spherical crowder over all $<p_{c}$ because rodlike crowders render a larger covolume than spherical crowders. Because the effects found upon including only volume exclusion cannot explain the experimental results of dextran 20-induced elongation of unfolded apoazurin, we next investigated a dextran 20 model that also provides effective attractive interactions.

\subsubsection{Structural Changes of Apoazurin with Which Crowders Interact} Attractively and under Chemical Denaturation.-We hypothesize the existence of nonspecific attractive interactions between the unfolded protein and crowders. For this, we tested two types of nonspecific attractive interactions with $\lambda=0.83$ and $\lambda=2.50$ (see Materials and Methods). $\lambda$ measures the attraction between a protein and crowder relative to the attraction between two Thr residues at $0 \mathrm{M}$ urea. $\lambda=0.83$ represents a weak attraction, and $\lambda=2.50$ represents a strong attraction.

We noticed that a box of spherical crowders with a weak attraction (DX20A-S at $\lambda=0.83$ ) cannot produce elongated ensembles of apoazurin in silico at room temperature with $k_{\mathrm{B}} T_{\mathrm{u}} / \varepsilon$ $=1.14$ in Figure 6 . The distribution of $\Delta$ for apoazurin, centered at $\Delta \approx 0.16$, is significantly narrower than the bulk condition over a wide range of $\phi_{\mathrm{c}}$. We note that, with a strong attraction at $\lambda=2.5$, there was a slight increase in $\Delta / \Delta_{\mathrm{B}}$ or $R_{\mathrm{g}} / R_{\mathrm{g}, \mathrm{B}}$ along $\phi_{\mathrm{c}}$ (Figure S6). However, $R_{\mathrm{g}} / R_{\mathrm{g}, \mathrm{B}}$ is still less than 1 when the $\mathrm{AR}$ of the crowder is less than 4.

This result leads us to a working hypothesis that, in addition to weak attractions, the crowders must be rodlike to stabilize an extended unfolded conformation of apoazurin. We showed that, in the system of weakly attractive crowders with a rodlike shape (DX20A- 
AR4), the distribution of $\Delta$ for the protein apoazurin under the chemically denatured condition becomes broad and comparable to that under the bulk condition in Figure 6 . Interestingly, the distribution of $\Delta$ for apoazurin shifts toward $\Delta=1$ and forms a large peak at $\Delta \approx 0.84$ at $\phi_{\mathrm{c}}=5 \%$ for DX20A-AR8 (Figure 7). This finding implies that more elongated conformations of apoazurin in chemically unfolded states are sampled in a system with the crowder model DX20A-AR8 than with the crowder model DX20A-AR4. However, the height and position of the peak shifts toward $\Delta=0$ with increasing $\phi_{\mathrm{c}}$. At a high $\phi_{\mathrm{c}}$ of $20 \%$, apoazurin adopts a compact configuration again (Figure 8). This feature was not observed for spherical crowders (DX20A-S) with a weakly attractive interaction with a protein at $\lambda=$ 0.83 (Figure S6).

\subsubsection{Both the Rodlike Shape of a Crowder and Attractive Interaction with a Protein Contribute to the Structural Changes of Apoazurin under Chemically} Denatured Conditions.-We evaluated $\Delta / \Delta_{\mathrm{B}}$ as well as $R_{\mathrm{g}} / R_{\mathrm{g}, \mathrm{B}}$ of chemically denatured apoazurin at varying ARs of crowders for a wide range of $\phi_{\mathrm{c}}$ in Figure 8. Overall, there is an increase in $\Delta / \Delta_{\mathrm{B}}$ when AR increases (Figure 8a). $\Delta \Delta_{\mathrm{B}}$ exceeds 1 at AR greater than 8 . Interestingly, $R_{\mathrm{g}} / R_{\mathrm{g}, \mathrm{B}}$ also exceeds 1 at the most extreme case of $\mathrm{AR}=16$ (Figure $8 \mathrm{~b}$ ) at $\phi_{\mathrm{c}}=$ $5 \%$. However, we noted that the increasing trends in $\Delta \Delta_{\mathrm{B}}$ or $\mathrm{R}_{\mathrm{g}} / \mathrm{R}_{\mathrm{g}, \mathrm{B}}$ are not uniform with $\phi_{\mathrm{c}}$ 's. When AR is less than 4 , the protein has the largest $\Delta$ and $\mathrm{R}_{\mathrm{g}}$ values at $\phi_{\mathrm{c}}=20 \%$ compared with the other $\phi_{\mathrm{c}}$ conditions. When $\mathrm{AR}$ is greater than 4 , the largest $\Delta$ and $R_{\mathrm{g}}$ values occur at $\phi_{\mathrm{c}}=5 \%$.

Such a crossover behavior at $\mathrm{AR}=4$ is contributed by the shape of the protein-crowder assembly (Figure 9). The unfolded ensemble of apoazurin conformations adopts a shape according to the available void formed by surrounding crowders (Figure 9a). A rodlike crowder with nonspecific attractive interactions provides an elongated surface where several regions of the protein can stick (Figure 9b). This particular geometry of rodlike crowder favors the formation of extended unfolded conformations of apoazurin. As several crowders stick to a protein, they tend to align themselves and restrict the available space for the protein. It appears that, at high $\phi_{\mathrm{c}}$, there is insufficient space to accommodate the volume of a large assembly of protein crowders. Therefore, the uptick of $\Delta \Delta_{\mathrm{B}}$ or $R_{\mathrm{g}} / R_{\mathrm{g}, \mathrm{B}}$ at high $\phi_{\mathrm{c}}$ is weaker than that at low $\phi_{\mathrm{c}}$.

\subsubsection{Geometry of Crowder-Induced Voids is Modulated by the Protein-}

Crowder Interactions. - Next, we investigated the geometry of voids, in terms of shape and size, formed by crowders of the various models modulated by the protein-crowder interactions. The voids are the depletion zones due to nonoverlapping volume exclusion between a protein and crowders. The distribution of the asphericity of the void $\Delta^{\text {void }}$ is virtually unchanged between spherical and rodlike crowders without attractive interactions (Figure 10a). Additionally, as $\phi_{\mathrm{c}}$ increases, the peaks of the distributions are unperturbed. These results indicate that, without attractive interactions, the variation in the shape effect from rodlike crowders has been averaged in the ensemble. However, when attractive interactions are added to the crowders, there is a clear shift rightward (toward higher asphericity) of the peak. The spread of the distributions increases with AR when the shape of a crowder becomes elongated (Figure 10b). These effects are greatest for $\phi_{c}=5 \%$. 
We show that the distribution of the size of a void $\left(R_{g}^{\text {void }}\right)$ depends on the volume fraction of crowders, the shape of a crowder, and interaction between the protein and crowder. To make a fair comparison between spherical and rodlike crowders, we plotted the distribution of $\left(R_{\mathrm{g}}^{\mathrm{void}}-R\right) / \sigma$ in Figure $10 \mathrm{c}, \mathrm{d}$, where $R$ is the radius of a sphere in a crowder model. The overall size of $\left(R_{\mathrm{g}}^{\mathrm{void}}-R\right) / \sigma$ is smaller for rodlike crowders (DX20-AR8) than spherical crowders (DX20-S) by roughly a third at each $\phi_{\mathrm{c}}$ when a protein and crowders interact through hard-core interactions (Figure 10c). However, with a weak attraction between a protein and crowders $(\lambda=0.83),\left(R_{\mathrm{g}}^{\mathrm{void}}-R\right) / \sigma$ is reduced by half across all crowder types (Figure 10d) compared to those in Figure 10c. The peaks in the distribution of $\left(R_{\mathrm{g}}^{\text {void }}-R\right) / \sigma$ for rodlike crowders (DX20AAR8) at all $\phi_{\mathrm{c}}$ nearly overlap at the same position around 10. This feature is consistent with the finding of a protein-crowder assembly in Figure 9.

\section{DISCUSSION}

This in silico investigation was initiated by the anomalous observation from NMR experiments that the addition of $180 \mathrm{~g} / \mathrm{L}$ of dextran 20 alters the ensemble of urea-unfolded apoazurin in the direction of an extended conformation. Initially, this observation contrasts the physical intuition of the excluded volume theory that proteins generally favor a collapsed, compact conformation in the presence of macromolecular crowders. ${ }^{57}$ This intuition is built on a hard-sphere crowder (or depletant) that exerts entropic depletion interactions, or the macromolecular crowding effect, ${ }^{4}$ on the protein of interest, that effectively makes the unfolded expanded states of a protein entropically unfavorable, thus favoring a collapsed, compact conformation over an unfolded, extended one. ${ }^{18}$ The strength of the macromolecular crowding effect grows with the volume fraction of crowders and is often strongest when the radius of the crowders is smaller than the $R_{\mathrm{g}}$ of the test protein. ${ }^{58}$ However, recent studies have shown that the sequence-dependent properties of a protein from protein crowders can offset the volume exclusion of protein crowders. ${ }^{24,59}$ The sequence-dependent interactions between a protein and protein crowder include chemically soft interactions such as hydrogen bonding, electrostatic interactions, or dispersion. In some cases, these chemical attractions that possibly stick a protein to the surface of crowders further form quinary structures and destabilize the native folded state..$^{28,60,61}$

Chemically inert crowders such as Ficoll 70, PEG, and dextrans do not share the same chemical surfaces as protein crowders. However, a spherocylinder model for dextrans that lacks atomistic details and interacts with a protein through hard-core potentials fails to explain the anomaly from experiments, unless we consider the effective attractions between a protein and dextrans. A recent study by Zhou et al. also suggests that synthetic crowders may attain strong attractions with intrinsically disordered proteins (IDPs) such that IDP polypeptides populate expanded configurations under crowded conditions. ${ }^{62}$ The underlying reasons may include soft repulsion between the protein and synthetic crowders, deepening the depletion in the potential of mean force. ${ }^{29,30}$ This physically soft aspect of an interaction (in contrast to chemically soft as mentioned above) is due to the compressibility of softmatter materials that does not exist in nonoverlapping hard spheres. It is possible that, in the 
mixture of urea and dextran, dextran becomes physically soft. This speculation is supported by a recent essay from Ferreira et al. ${ }^{26}$ that, in the mixture of osmolyte and a macromolecular crowder, the solvent properties may not always be additive, depending on the composition of the mixture. We speculate that, at $6 \mathrm{M}$ urea, not only the solventmediated interactions between amino acids in the protein are weakened, leading to unfolding, but dextrans also become softened to trap unfolded apoazurin conformations. This scenario can be imagined as spaghetti trapped by a fork. This "fork-spaghetti" mechanism may be facilitated by the nonuniform length of the branches of dextran. ${ }^{63}$ The geometric feature of branched dextrans is at odds with the dimensions of a linear dextran model, ${ }^{12}$ especially when the molecular weight increases. ${ }^{22}$ Note-worthily, the behavior of branched polymers (dextran) is distinct from those of linear polymers (PEG) with a similar molecular weight shown by Soranno et al. ${ }^{64}$ who investigates the macromolecular crowding effect on the compaction of intrinsically disorder proteins (IDPs). When large PEG polymers reach a semi dilute region, they unwind and overlap one another at a moderate volume fraction of $10 \%$. The monomers of overlapping PEG polymers still exert volume exclusion effect on the compaction of IDPs. However, the strength of volume exclusion from PEG monomers is not as strong as that of nonoverlapping PEG polymers as predicted from the scaled particle theory. ${ }^{57}$

Notably, we showed that the effective attraction alone is not sufficient to bias the ensemble of unfolded apoazurin toward an elongated one as the shape of the crowder must be cylindrical with a sufficiently large AR. With spherical crowders containing either weak or strong effective attraction, the enhanced depletion interactions still favor a compact unfolded apoazurin configuration (Figure 8b). This contrasts the findings from Zhou's work in which peptide model samples with both extended and compact conformations interacted strongly with spherical crowders. ${ }^{62}$ We speculate that the biphasic distribution might change if the length of the simulation is increased or if a more efficient sampling scheme is introduced. Because both the aspherical shape of a crowder and its effective attraction with a protein increase the correlation time in the simulations, we ensured that our conclusions are independent of the sampling scheme in our simulations. Our results at a volume fraction of $5 \%$ for the DX20A-AR8 model at $\lambda=0.83$ do not depend on the initial unfolded conformation of apoazurin. The temporal evolution of $\Delta$ is shown in Figure 11 at the early stage of two canonical simulations: one starting from a compact unfolded conformation and the other from an extended unfolded conformation. In the first case, $\Delta$ fluctuates and rearranges initially, and then it increases as time increases. In the second case, the conformation becomes compact from a stretched one, and then it rearranges to a have a less extended shape. Thus, the initial conformation is not the reason why the simulations favor the formation of extended conformations with crowders and nonspecific attractive interactions.

To explain our in silico results, the elongated shape of a crowder must break the symmetry of the isotropic direction of the depletion force, creating an anisotropic force that sandwiches the backbone of the protein. We found that the elongated conformation of apoazurin is stabilized by a bundle of rodlike crowders in the presence of effective attraction. The rodlike crowders are still free to rotate, and there is no evidence of an isotropic-nematic transition in our system. In the absence of effective attraction, the repulsive cylinders do not 
self-assemble into nematic states. To reach a nematic state for rodlike crowders with an AR of $5, \phi_{c}$ typically must exceed $40 \% .{ }^{65}$ In fact, hard spherocylinders contribute to greater compactness of apoazurin because of an increased covolume compared with a volumeconserving hard sphere. ${ }^{4}$ The difference here is the effective attraction of apoazurin and several rodlike crowders that collectively form a bundle. The bundles formed around elongated apoazurin maximize the volume accessible to the other crowders (not in a bundle), thus maximizing the total entropy of the system. We showed that elongated apoazurin appears at low $\phi_{\mathrm{c}}=5 \%$, but not at high $\phi_{\mathrm{c}}=20 \%$; the latter was observed in in vitro experiments. This difference may be due to the increase in excluded volume interactions between hard spherocylinders instead of the spherocylinder-apoazurin interactions, as the volume fraction of spherocylinders increases. To induce the formation of significant bundles at high $\phi_{\mathrm{c}}$, the concentration of apoazurin in a binary mixture of spherocylinder and apoazurin should be increased.

\section{CONCLUSIONS}

New understanding in science generally begins when theoretical expectations and new experimental findings do not agree. Here, the intriguing NMR discovery showing elongated urea-unfolded states in the presence of dextran goes against intuition from conventional macromolecular crowding theory and previous experimental findings. With very little clues from experimental data, we have employed coarse-grained modeling to investigate the underlying mechanism. Even though we cannot definitively exclude other possible explanations for the trends found in the experiments, our work denotes the importance of both crowder shapes and solvent-mediated interactions in manipulating the geometry of a protein. These two properties are pivotal to understand the full complexity of proteins in the cytoplasm. Furthermore, this newfound understanding can potentially be developed into a design principle for probes to accurately measure crowding volume fraction, shapes, and interactions locally in a subcellular environment.

\section{Supplementary Material}

Refer to Web version on PubMed Central for supplementary material.

\section{ACKNOWLEDGMENTS}

We thank Alexander Christiansen for providing apoazurin and Birgit Köhn for technical assistance. We acknowledge the funding support from the National Science Foundation (MCB1412532 and OAC-1531814) and the computational resources from the Center for Advanced Computing and Data Science (CACDS) and Research Computing Center (RCC) at the University of Houston. M.S.C. thanks Yossi Eliaz for his intellectual discussion. A.G.G. is supported by a fellowship from the Houston Area Biophysics Program (T32 GM008280). P.W.-S. acknowledges funding from Knut and Alice Wallenberg Foundation and the Swedish Research Council. M.K. acknowledges financial support from the Young Scholar Fund of the Universität Konstanz.

\section{REFERENCES}

(1). Ellis RJ; Minton AP Join the crowd. Nature 2003, 425, 27-28. [PubMed: 12955122]

(2). Link AJ; Robison K; Church GM Comparing the predicted and observed properties of proteins encoded in the genome ofEscherichia coli K-12. Electrophoresis 1997, 18, 1259-1313. [PubMed: 9298646] 
(3). Luby-Phelps K. Cytoarchitecture and physical properties of cytoplasm: Volume, viscosity, diffusion, intracellular surface area. Int. Rev. Cytol 1999, 192, 189-221.

(4). Minton AP Excluded volume as a determinant of macromolecular structure and reactivity. Biopolymers 1981, 20, 2093-2120.

(5). Venturoli D; Rippe B. Ficoll and dextran vs. globular proteins as probes for testing glomerular permselectivity: Effects of molecular size, shape, charge, and deformability. Am. J. Physiol. Renal. Physiol 2005, 288, F605-F613. [PubMed: 15753324]

(6). Homouz D; Perham M; Samiotakis A; Cheung MS; Wittung-Stafshede P. Crowded, cell-like environment induces shape changes in aspherical protein. Proc. Natl. Acad. Sci. U.S.A 2008, 105, 11754-11759. [PubMed: 18697933]

(7). Christiansen A; Wang Q; Samiotakis A; Cheung MS; Wittung-Stafshede P. Factors Defining Effects of Macromolecular Crowding on Protein Stability: An in Vitro/in Silico Case Study Using Cytochromec. Biochemistry 2010, 49, 6519-6530. [PubMed: 20593812]

(8). Dhar A; Samiotakis A; Ebbinghaus S; Nienhaus L; Homouz D; Gruebele M; Cheung MS Structure, function, and folding of phosphoglycerate kinase are strongly perturbed by macromolecular crowding. Proc. Natl. Acad. Sci. U.S.A 2010, 107, 17586-17591. [PubMed: 20921368]

(9). Gupta AN; van der Maarel JRC Compaction of plasmid DNA by macromolecular crowding. Macromolecules 2017, 50, 1666-1671.

(10). Zinchenko A; Berezhnoy NV; Chen Q; Nordenskiöld L. Compaction of single-molecule megabase-long chromatin under the influence of macromolecular crowding. Biophys. J 2018, 114, 2326-2335. [PubMed: 29729833]

(11). Christiansen A; Wittung-Stafshede P. Quantification of excluded volume effects on the folding landscape of Pseudomonas aeruginosa Apoazurin in vitro. Biophys. J 2013, 105, 1689-1699. [PubMed: 24094410]

(12). Sasahara K; McPhie P; Minton AP Effect of dextran on protein stability and conformation attributed to macromolecular crowding. J. Mol. Biol 2003, 326, 1227-1237. [PubMed: 12589765]

(13). Christiansen A; Wittung-Stafshede P. Synthetic crowding agent dextran causes excluded volume interactions exclusively to tracer protein apoazurin. FEBS Lett. 2014, 588, 811-814. [PubMed: 24491997]

(14). Yu I; Mori T; Ando T; Harada R; Jung J; Sugita Y; Feig M. Biomolecular interactions modulate macromolecular structure and dynamics in atomistic model of a bacterial cytoplasm. eLife 2016, 5, e19274.

(15). McGuffee SR; Elcock AH Diffusion, Crowding \& Protein Stability in a Dynamic Molecular Model of the Bacterial Cytoplasm. PLoS Comput. Biol 2010, 6, e1000694.

(16). Dong H; Qin S; Zhou H-X Effects of macromolecular crowding on protein conformational changes. PLoS Comput. Biol 2010, 6, e1000833.

(17). Qin S; Minh DDL; McCammon JA; Zhou H-X Method to predict crowding effects by postprocessing molecular dynamics trajectories: Application to the flap dynamics of HIV-1 protease. J. Phys. Chem. Lett 2010, 1, 107-110. [PubMed: 20228897]

(18). Cheung MS; Klimov D; Thirumalai D. Molecular crowding enhances native state stability and refolding rates of globular proteins. Proc. Natl. Acad. Sci. U.S.A 2005, 102, 4753-4758. [PubMed: 15781864]

(19). Mittal J; Best RB Dependence of protein folding stability and dynamics on the density and composition of macromolecular crowders. Biophys. J 2010, 98, 315-320. [PubMed: 20338853]

(20). Wang Q; Cheung MS A Physics-Based Approach of Coarse-Graining the Cytoplasm of Escherichia coli (CGCYTO). Biophys. J 2012, 102, 2353-2361. [PubMed: 22677389]

(21). Kudlay A; Cheung MS; Thirumalai D. Influence of the shape of crowding particles on the structural transitions in a polymer. J. Phys. Chem. B 2012, 116, 8513-8522. [PubMed: 22616622]

(22). Granath KA Solution properties of branched dextrans. J. Colloid Sci 1958, 13, 308-328. 
(23). Homouz D; Stagg L; Wittung-Stafshede P; Cheung MS Macromolecular Crowding Modulates Folding Mechanism of $\alpha / \beta$ Protein Apoflavodoxin. Biophys. J 2009, 96, 671-680. [PubMed: 19167312]

(24). Jiao M; Li H-T; Chen J; Minton AP; Liang Y. Attractive protein-polymer interactions markedly alter the effect of macromolecular crowding on protein association equilibria. Biophys. J 2010, 99, 914-923. [PubMed: 20682270]

(25). Senske M; Törk L; Born B; Havenith M; Herrmann C; Ebbinghaus S. Protein Stabilization by Macromolecular Crowding through Enthalpy Rather Than Entropy. J. Am. Chem. Soc 2014, 136, 9036-9041. [PubMed: 24888734]

(26). Ferreira LA; Uversky VN; Zaslavsky BY Role of solvent properties of water in crowding effects induced by macromolecular agents and osmolytes. Mol. BioSyst 2017, 13, 2551-2563. [PubMed: 29018861]

(27). Gorensek-Benitez AH; Smith AE; Stadmiller SS; Perez Goncalves GM; Pielak GJ Cosolutes, crowding, and protein folding kinetics. J. Phys. Chem. B 2017, 121, 6527-6537. [PubMed: 28605189]

(28). Mu X; Choi S; Lang L; Mowray D; Dokholyan NV; Danielsson J; Oliveberg M. Physicochemical code for quinary protein interactions inEscherichia coli. Proc. Natl. Acad. Sci. U.S.A 2017, 114, E4556-E4563. [PubMed: 28536196]

(29). Rovigatti L; Gnan N; Parola A; Zaccarelli E. How soft repulsion enhances the depletion mechanism. Soft Matter 2015, 11, 692-700. [PubMed: 25428843]

(30). Sapir L; Harries D. Origin of enthalpic depletion forces. J. Phys. Chem. Lett 2014, 5, 1061-1065. [PubMed: 26274449]

(31). Sapir L; Harries D. Is the depletion force entropic? Molecular crowding beyond steric interactions. Curr. Opin. Colloid Interface Sci 2015, 20, 3-10.

(32). Adman ET, Copper protein structures. Metalloproteins: Structural Aspects; Elsevier, 1991; Vol. 42, pp 145-197.

(33). Wilson CJ; Apiyo D; Wittung-Stafshede P. Solvation of the folding-transition state in Pseudomonas aeruginosa azurin is modulated by metal: Solvation of azurin's folding nucleus. Protein Sci. 2006, 15, 843-852. [PubMed: 16522792]

(34). Zong C; Wilson CJ; Shen T; Wolynes PG; Wittung-Stafshede P. $\varphi$-Value Analysis of Apo-Azurin Folding: Comparison between Experiment and Theory ${ }^{\dagger}$. Biochemistry 2006, 45, 6458-6466. [PubMed: 16700556]

(35). Wang Q; Christiansen A; Samiotakis A; Wittung-Stafshede P; Cheung MS Comparison of chemical and thermal protein denaturation by combination of computational and experimental approaches. II. J. Chem. Phys 2011, 135, 175102.

(36). Humphrey W; Dalke A; Schulten K. VMD: Visual molecular dynamics. J. Mol. Graphics Modell 1996, 14, 33-38.

(37). Kabsch W; Sander C. Dictionary of protein secondary structure: Pattern recognition of hydrogenbonded and geometrical features. Biopolymers 1983, 22, 2577-2637. [PubMed: 6667333]

(38). Delaglio F; Grzesiek S; Vuister GW; Zhu G; Pfeifer J; Bax A. NMRPipe: A multidimensional spectral processing system based on UNIX pipes. J. Biomol. NMR 1995, 6, 277-293. [PubMed: 8520220]

(39). Johnson BA; Blevins RA NMR View: A computer program for the visualization and analysis of NMR data. J. Biomol. NMR 1994, 4, 603-614. [PubMed: 22911360]

(40). Jones JA; Wilkins DK; Smith LJ; Dobson CM Characterisation of protein unfolding by NMR diffusion measurements. J. Biomol. NMR 1997, 10, 199-203.

(41). Berger S; Braun S. 200 and More NMR Experiments: A Practical Course; Wiley-Vch: Weinheim, 2004.

(42). Wilkins DK; Grimshaw SB; Receveur V; Dobson CM; Jones JA; Smith LJ Hydrodynamic Radii ofNative and Denatured Proteins Measured by Pulse Field Gradient NMR Techniques ${ }^{\dagger}$. Biochemistry 1999, 38, 16424-16431. [PubMed: 10600103]

(43). Armstrong JK; Wenby RB; Meiselman HJ; Fisher TC The hydrodynamic radii of macromolecules and their effect on red blood cell aggregation. Biophys. J 2004, 87, 4259-4270. [PubMed: 15361408] 
(44). Cheung MS; Finke JM; Callahan B; Onuchic JN Exploring the interplay between topology and secondary structural formation in the protein folding problem. J. Phys. Chem. B 2003, 107, 11193-11200.

(45). Bryngelson JD; Wolynes PG Spin glasses and the statistical mechanics of protein folding. Proc. Natl. Acad. Sci. U.S.A 1987, 84, 7524-7528. [PubMed: 3478708]

(46). Onuchic JN; Luthey-Schulten Z; Wolynes PG Theory of protein folding: The energy landscape perspective. Annu. Rev. Phys. Chem 1997, 48, 545-600. [PubMed: 9348663]

(47). Sheinerman FB; Brooks CL III Calculations on folding of segment B1 of streptococcal protein G 1 1Edited by F. Cohen. J. Mol. Biol 1998, 278, 439-456. [PubMed: 9571063]

(48). Samiotakis A; Cheung MS Folding dynamics of Trp-cage in the presence of chemical interference and macromolecular crowding. I. J. Chem. Phys 2011, 135, 175101.

(49). Case DA; Darden TA; Cheatham TE; Simmerling CL; Wang J; Duke RE; Luo R; Crowley M; Walker RC; Zhang W; et al. AMBER 10; University of California: San Francisco, 2008.

(50). Veitshans T; Klimov D; Thirumalai D. Protein folding kinetics: timescales, pathways and energy landscapes in terms of sequence-dependent properties. Fold. Des 1997, 2, 1-22. [PubMed: 9080195]

(51). Sanbonmatsu KY; García AE Structure of Met-Enkephalin in explicit aqueous solution using replica exchange molecular dynamics. Proteins: Struct., Funct. Bioinf 2002, 46, 225-234.

(52). Sugita Y; Okamoto Y. Replica-exchange molecular dynamics method for protein folding. Chem. Phys. Lett 1999, 314, 141-151.

(53). Dima RI; Thirumalai D. Asymmetry in the Shapes of Folded and Denatured States of Proteinsf. J. Phys. Chem. B 2004, 108, 6564-6570.

(54). Barber CB; Dobkin DP; Huhdanpaa H. The quickhull algorithm for convex hulls. ACM Trans. Math Software 1996, 22, 469-483.

(55). Dyson HJ; Wright PE How does your protein fold? Elucidating the apomyoglobin folding pathway. Acc. Chem. Res 2017, 50, 105-111. [PubMed: 28032989]

(56). Kovermann M; Rogne P; Wolf-Watz M. Protein dynamics and function from solution state NMR spectroscopy. Q. Rev. Biophys 2016, 49, E6. [PubMed: 27088887]

(57). Minton AP Models for excluded volume interaction between an unfolded protein and rigid macromolecular cosolutes: Macromolecular crowding and protein stability revisited. Biophys. J 2005, 88, 971-985. [PubMed: 15596487]

(58). Kang H; Pincus PA; Hyeon C; Thirumalai D. Effects of macromolecular crowding on the collapse of biopolymers. Phys. Rev Lett 2015, 114, 068303.

(59). Miklos AC; Sarkar M; Wang Y; Pielak GJ Protein crowding tunes protein stability. J. Am. Chem. Soc 2011, 133, 7116-7120. [PubMed: 21506571]

(60). Gnutt D; Timr S; Ahlers J; Konig B; Manderfeld E; Heyden M; Sterpone F; Ebbinghaus S. Stability Effect of Quinary Interactions Reversed by Single Point Mutations. J. Am. Chem. Soc 2019, 141, 4660-4669. [PubMed: 30740972]

(61). Cohen RD; Pielak GJ Electrostatic contributions to protein quinary structure. J. Am. Chem. Soc 2016, 138, 13139-13142. [PubMed: 27676610]

(62). Banks A; Qin S; Weiss KL; Stanley CB; Zhou H-X Intrinsically Disordered Protein Exhibits Both Compaction and Expansion under Macromolecular Crowding. Biophys. J 2018, 114, 1067 1079. [PubMed: 29539394]

(63). Ebert KH; Brosche M. Origin of branches in native dextrans. Biopolymers 1967, 5, 423-430. [PubMed: 6043409]

(64). Soranno A; Koenig I; Borgia MB; Hofmann H; Zosel F; Nettels D; Schuler B. Single-molecule spectroscopy reveals polymer effects of disordered proteins in crowded environments. Proc. Natl. Acad. Sci. U.S.A 2014, 111, 4874-4879. [PubMed: 24639500]

(65). Lago S; Cuetos A; Martínez-Haya B; Rull LF Crowding effects in binary mixtures of rod-like and spherical particles. J. Mol. Recognit 2004, 17, 417-425. [PubMed: 15362100] 
(a)

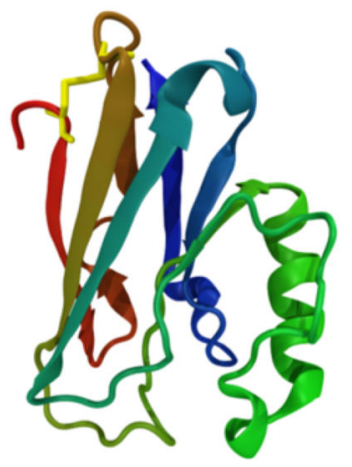

(b)

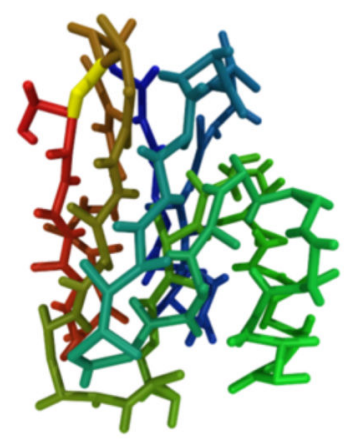

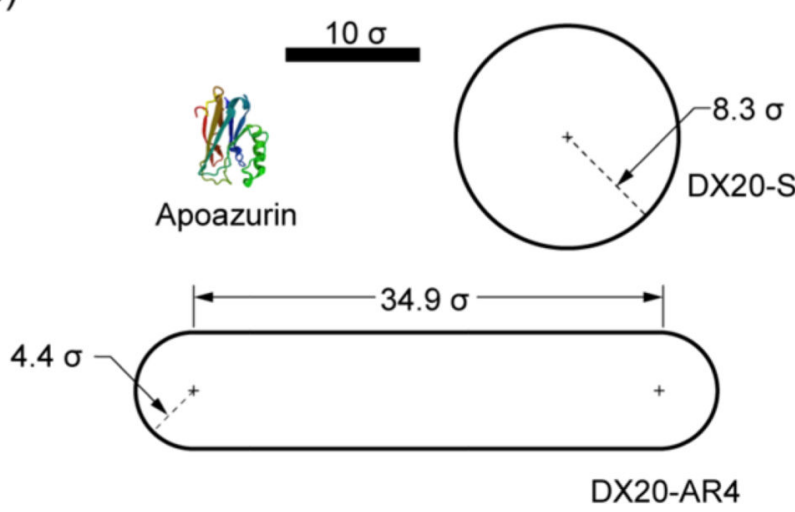

Figure 1.

(a) Representations of the folded structure of apoazurin (PDB ID: 1E65). The folded structure is illustrated in a cartoon representation and a side-chain $C_{a}$ representation. These protein illustrations were produced using VMD. ${ }^{36}$ The algorithm $\mathrm{DSSP}^{37}$ was used to assign the secondary structures. The residues that form the disulfide bond are shown in yellow. (b) Illustrations of two models for dextran 20 with the same volume: a spherical model (DX20S) and a spherocylinder model with an aspect ratio (AR) of 4 (DX20-AR4). As reference, the folded structure of apoazurin is shown. The reduced unit of length is $\sigma$. 


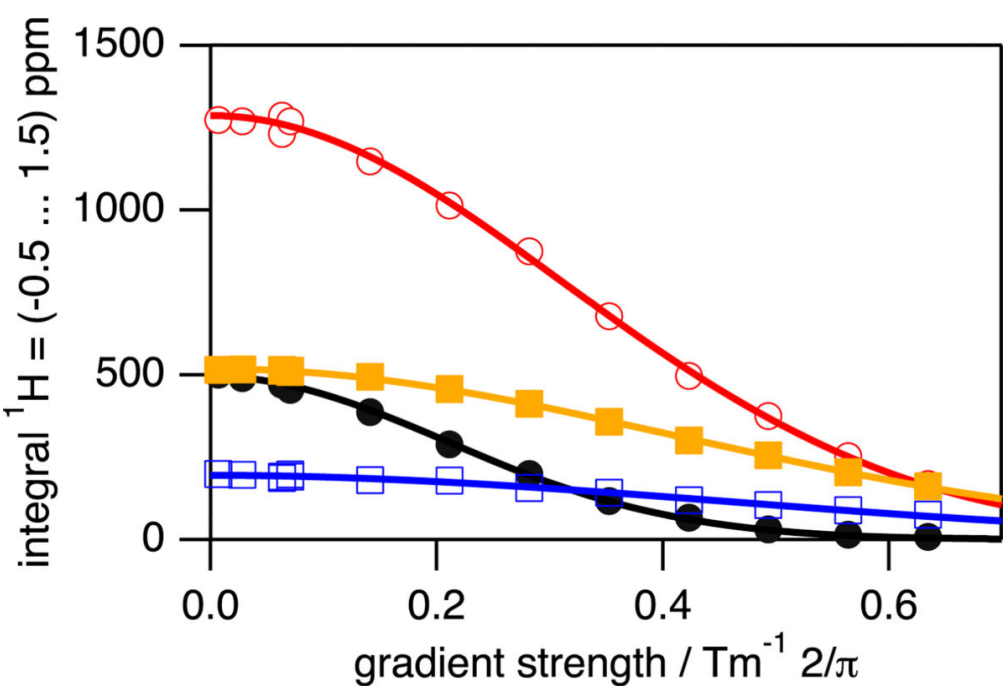

Figure 2.

PFG-NMR based diffusion profiles obtained for native apoazurin in absence (colored in black, closed circles) and in the presence of $c^{\mathrm{d} 20}=100 \mathrm{~g} / \mathrm{L}$ (colored in red, open circles), $c^{\mathrm{d} 20}=150 \mathrm{~g} / \mathrm{L}$ (colored in orange, closed rectangles), and $c^{\mathrm{d} 20}=200 \mathrm{~g} / \mathrm{L}$ (colored in blue, open rectangles). The straight lines are fits of eq 1 to the experimental data. All data were recorded at $T=298 \mathrm{~K}$. Particular gradient strengths have been repeated $(1,10,40$, and $70 \%$ related to maximum gradient strength possible). 


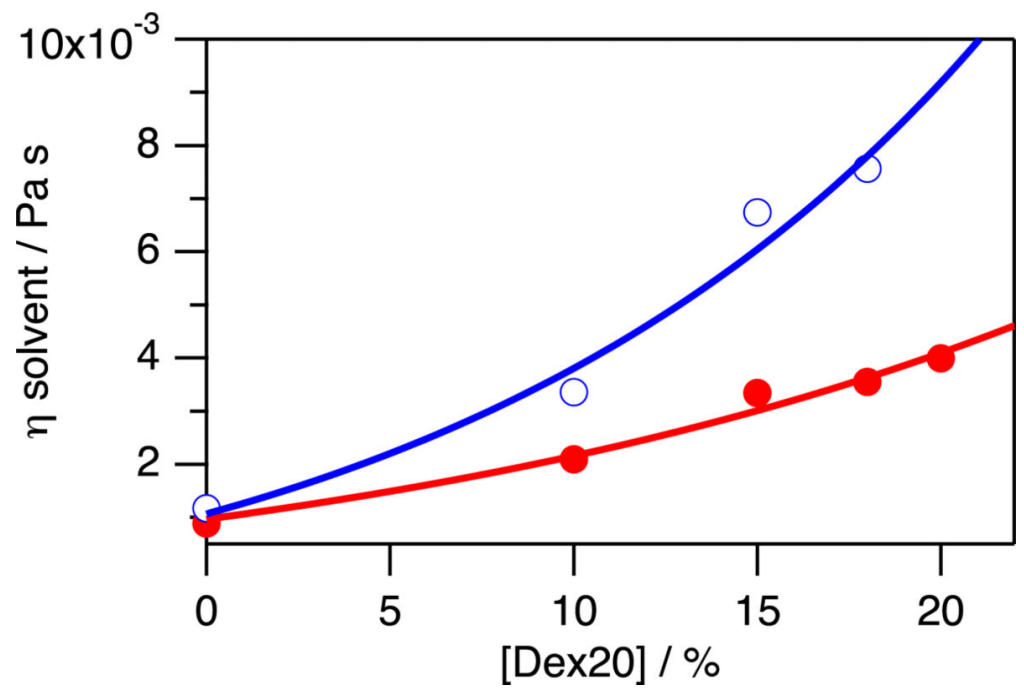

Figure 3.

Viscosity $\eta$ of the solvent depends on the concentrations of dextran 20 and urea (absence of urea: closed circles, colored in red; presence of $c=6.8 \mathrm{M}$ urea: open circles, colored in blue) and has been calculated by determining the diffusion of dioxane and dextran 20 and eq 2 at $T=298 \mathrm{~K}$. The straight lines have been computed by assuming a polynomial function to guide the eye. Error bars have been included. They are too small to be seen. They have been calculated based on the standard deviation of the diffusion coefficient obtained by fitting eq 1 to the diffusion data. This error of the diffusion coefficient has been used to determine the error of $r_{H}$ by using eq 2 . 


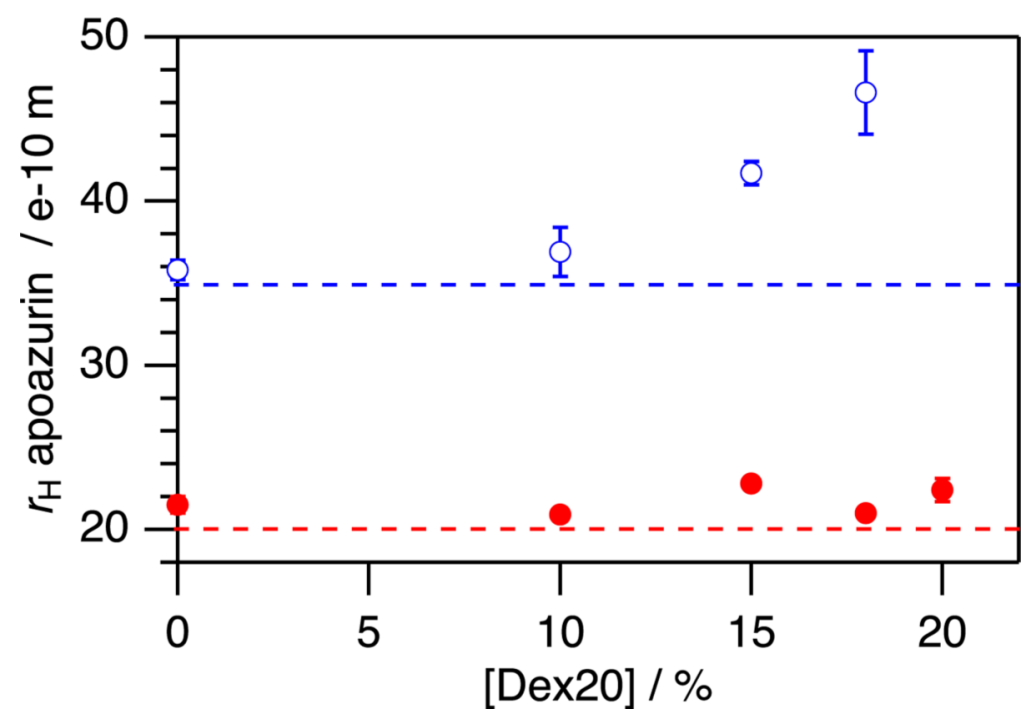

Figure 4.

Hydrodynamic radius $r_{\mathrm{H}}$ of apoazurin depends on the macromolecular crowding conditions used. Whereas native apoazurin does not show a dependence on the concentration of dextran 20 (closed circles, colored in red), denatured apoazurin increases in the hydrodynamic dimension by increasing the concentration of dextran 20 (open circles, colored in blue). The horizontal lines (dotted mode) show the hydrodynamic radii that are theoretically expected for a globular folded protein (colored in red) and a denatured polypeptide chain of that size (colored in blue) according to ref 42 . All the data were recorded at $T=298 \mathrm{~K}$. Error bars have been included (and seen). The calculation has been done in the same way we did Figure 3. 
(a)

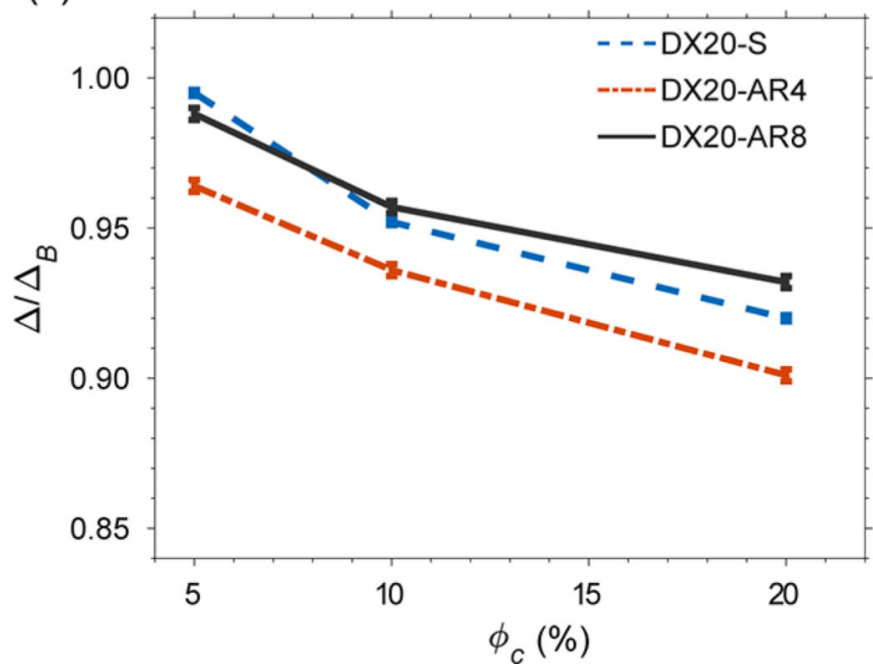

(b)

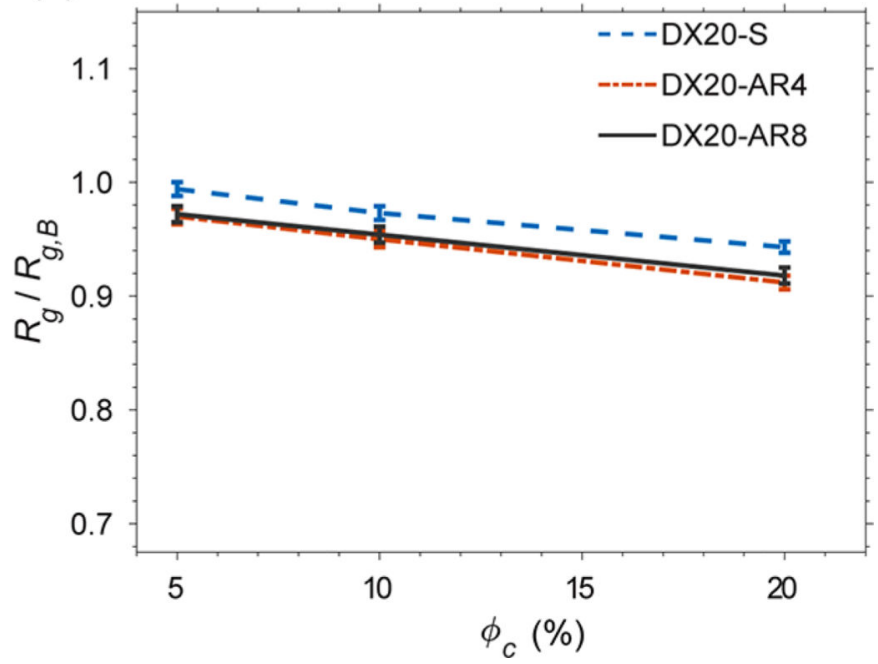

Figure 5.

(a) Average asphericity $(\Delta)$ of the protein apoazurin in varying shapes of crowders normalized by the average asphericity $\left(\Delta_{\mathrm{B}}\right)$ of apoazurin under the bulk condition against the volume fractions of crowders $\left(\phi_{\mathrm{c}}\right)$. (b) Average radius of gyration $\left(R_{\mathrm{g}}\right)$ of apoazurin normalized by the average radius of gyration under the bulk condition against $\phi_{\mathrm{c}}$. The interactions between the protein apoazurin and crowders are repulsive. The concentration of urea is $c^{\text {urea }}=6 \mathrm{M}$. The temperature is $k_{\mathrm{B}} T_{\mathrm{u}} / \mathcal{E}=1.14$, corresponding to room temperature (see Materials and Methods) for all conditions. Error bars are included. 


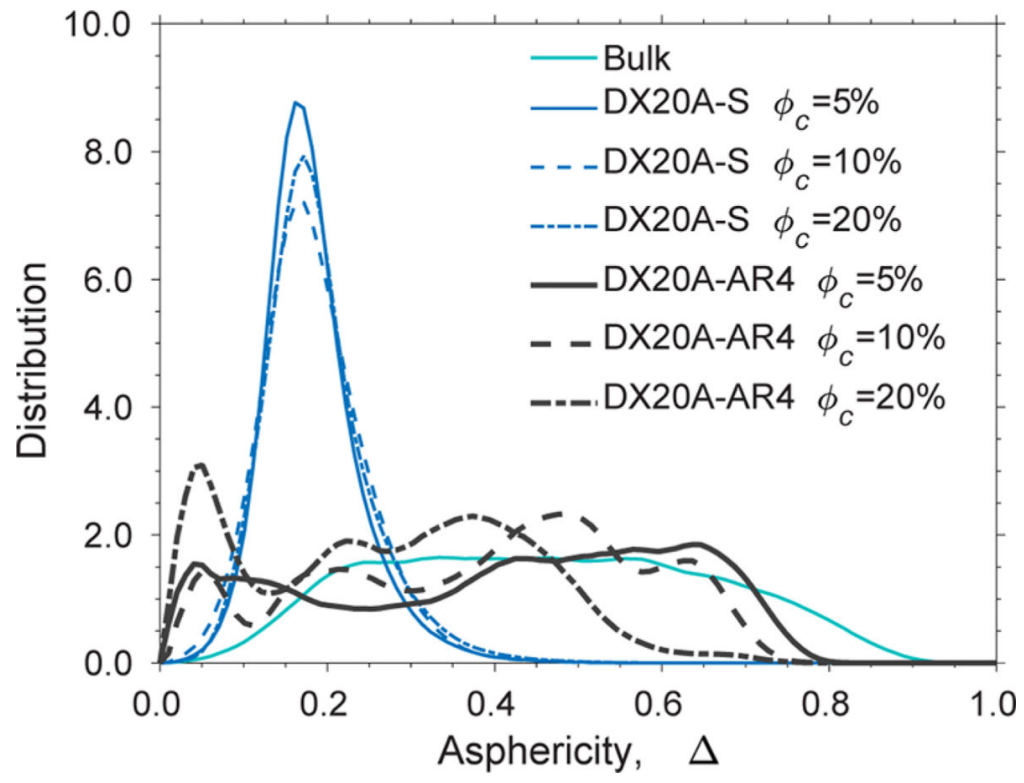

Figure 6.

Distribution of the asphericity $(\Delta)$ at $c^{\mathrm{drea}}=6 \mathrm{M}$ in bulk, with DX20A-S, and with DX20AAR4 with a strength of attractive interactions $\lambda=0.83$ at a wide range of volume fraction of crowders $\left(\phi_{\mathrm{c}}\right)$. The temperature is $k_{\mathrm{B}} T_{\mathrm{u}} / \varepsilon=1.14$. 


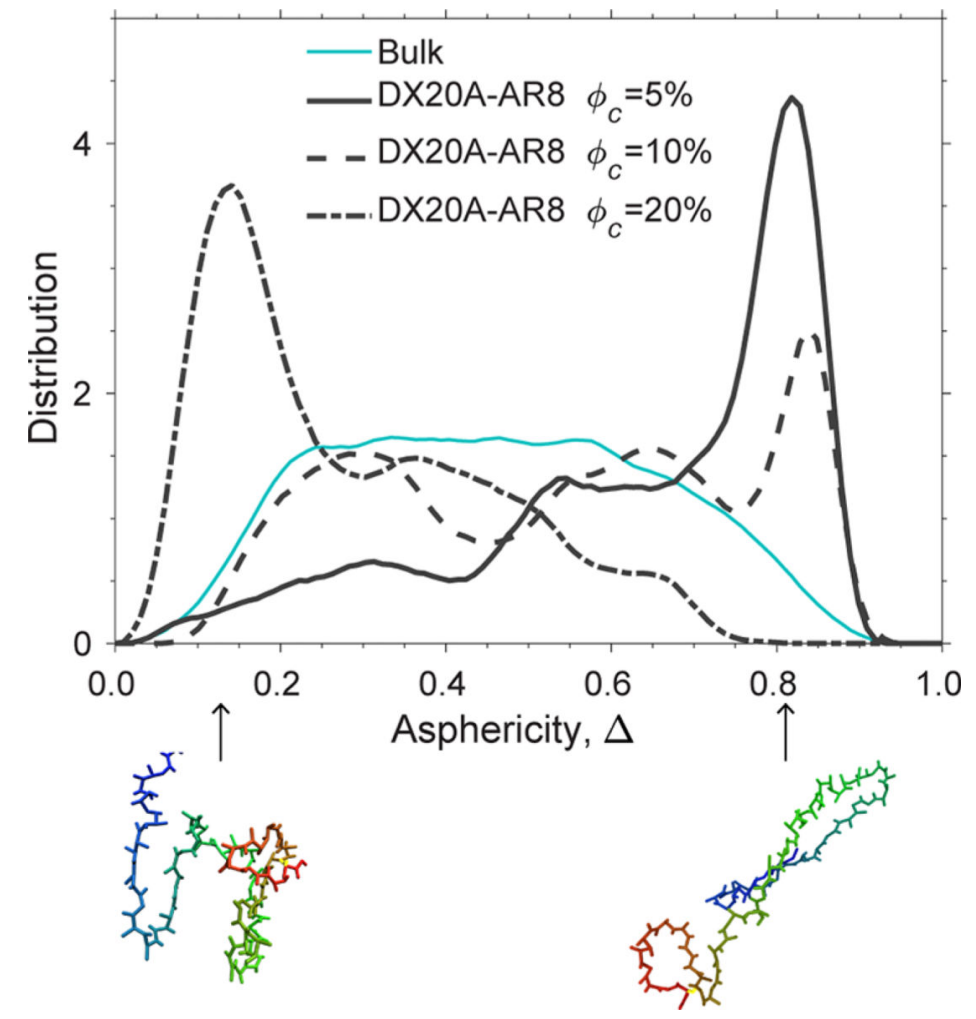

Figure 7.

Distribution of the asphericity $(\Delta)$ for chemically unfolded apoazurin at $6 \mathrm{M}$ urea in bulk and in a system of weakly attractive crowders (DX20A-AR8 at $\lambda=0.83$ ) over several volume fractions $\left(\phi_{\mathrm{c}}\right)$. The temperature for all conditions is $k_{\mathrm{B}} T_{\mathrm{u}} / \varepsilon=1.14$. Two representative structures are shown: at the left bottom with $\Delta=0.14\left(\phi_{\mathrm{c}}=20 \%\right)$ and the right bottom with $\Delta=0.83\left(\phi_{\mathrm{c}}=5 \%\right)$. 
(a)

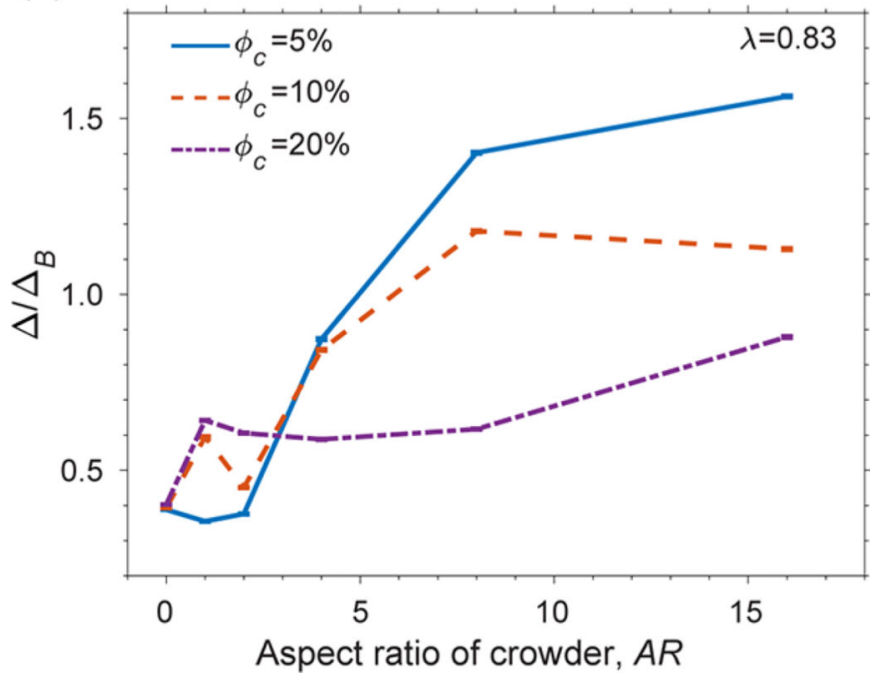

(b)

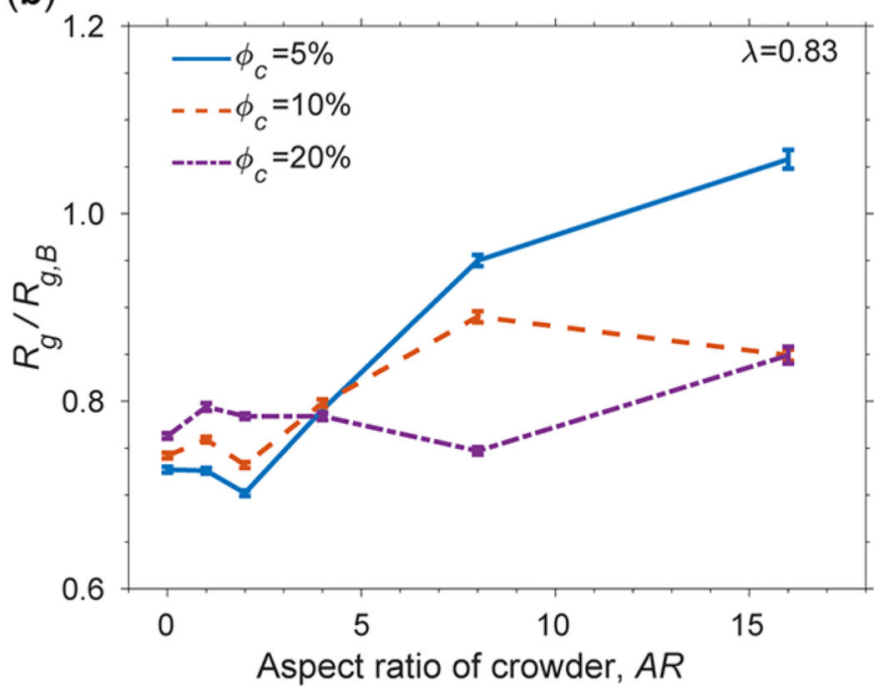

Figure 8.

(a) Ratio of the average of the asphericity $(\Delta)$ under a specific condition and average of the asphericity $\left(\Delta_{\mathrm{B}}\right)$ under the bulk condition. (b) Ratio of the average of the radius of gyration $\left(R_{\mathrm{g}}\right)$ under a specific condition and average of the radius of gyration $\left(R_{\mathrm{g}, \mathrm{B}}\right)$ under the bulk condition. These ratios are shown as a function of the AR of crowders. The concentration of urea is $6 \mathrm{M}$, and the temperature is $k_{\mathrm{B}} T_{\mathrm{u}} / \varepsilon=1.14$ for all conditions. Error bars are included. 
(a)

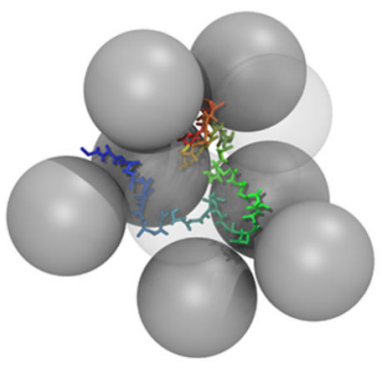

(b)

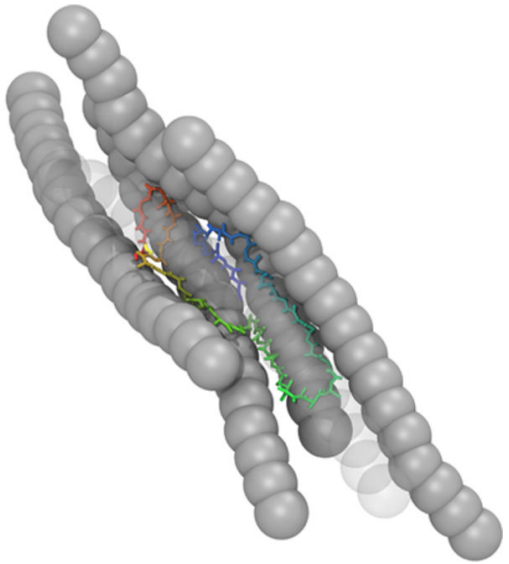

Figure 9.

Snapshots of an exemplarily unfolded structure of apoazurin and the void between the protein and closest crowders for (a) spherical crowders (DX20A-S) (b) and rodlike crowders (DX20-AAR8) at $\phi_{\mathrm{c}}=5 \%$ and at $c^{\text {urea }}=6 \mathrm{M}$ with weakly attractive interactions $(\lambda=0.83)$. 
(a)

(c)

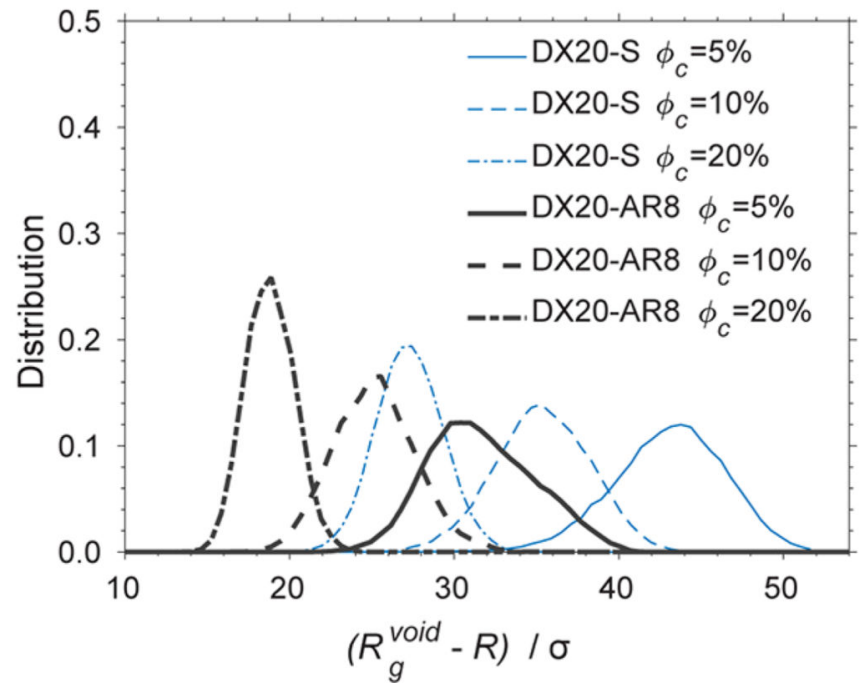

Figure 10. (b)

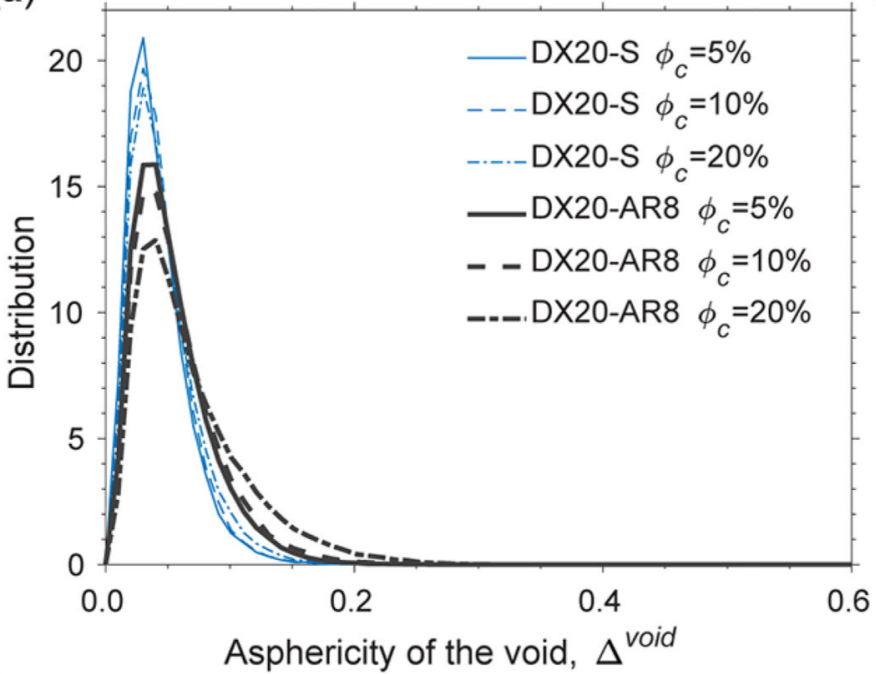

(d)

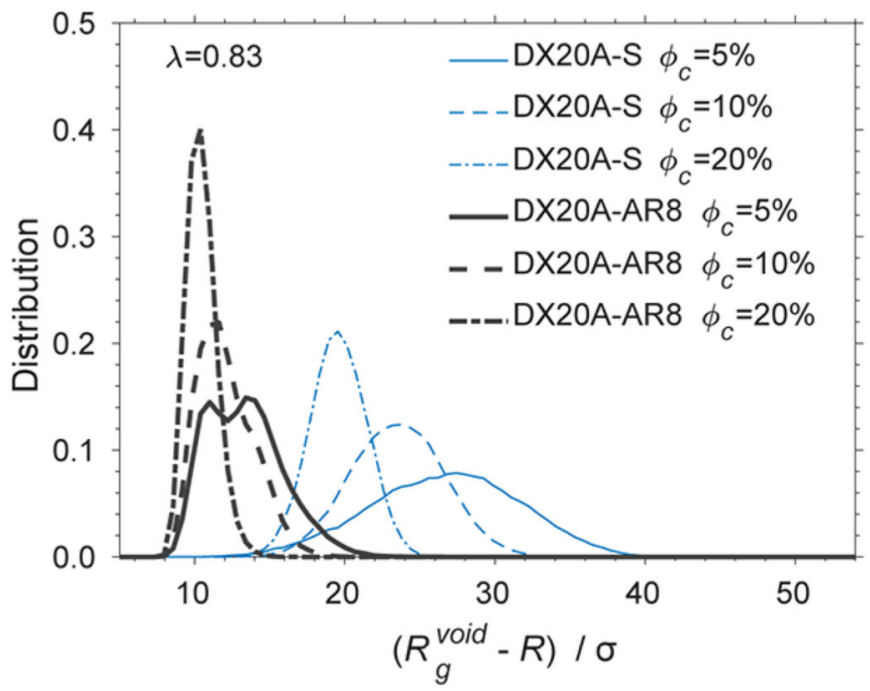

Distribution of the asphericity of the void ( $\Delta^{\text {void }}$ ) between the protein and crowders with (a) steric repulsive interactions and (b) nonspecific attractive interactions between the protein and crowders. The distribution of the radius of gyration of the $\operatorname{void}\left(R_{\mathrm{g}}^{\mathrm{void}}-R\right) / \sigma$ between the protein and crowders with (c) steric repulsive interactions and (d) weakly attractive interactions between the protein and crowders. 
(a)

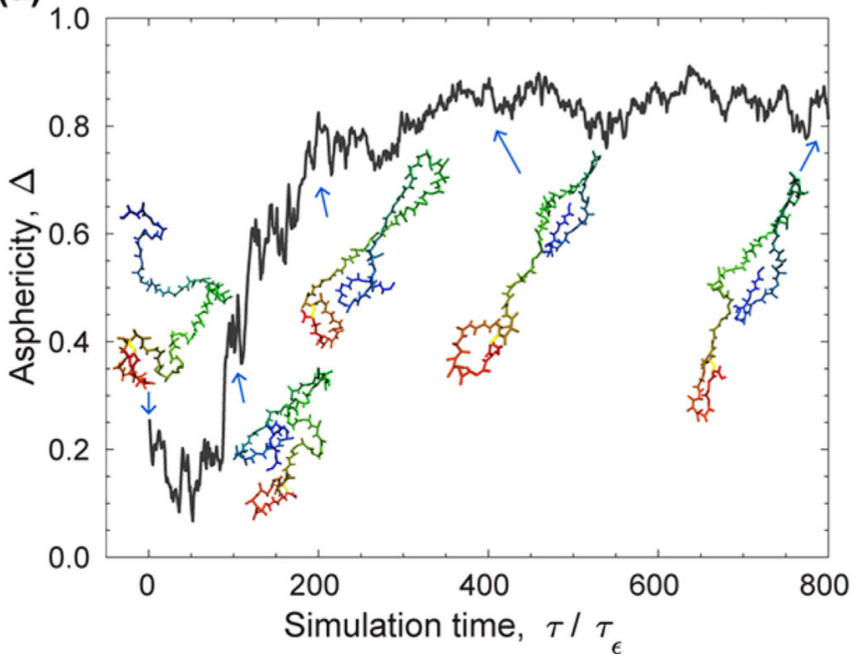

(b)

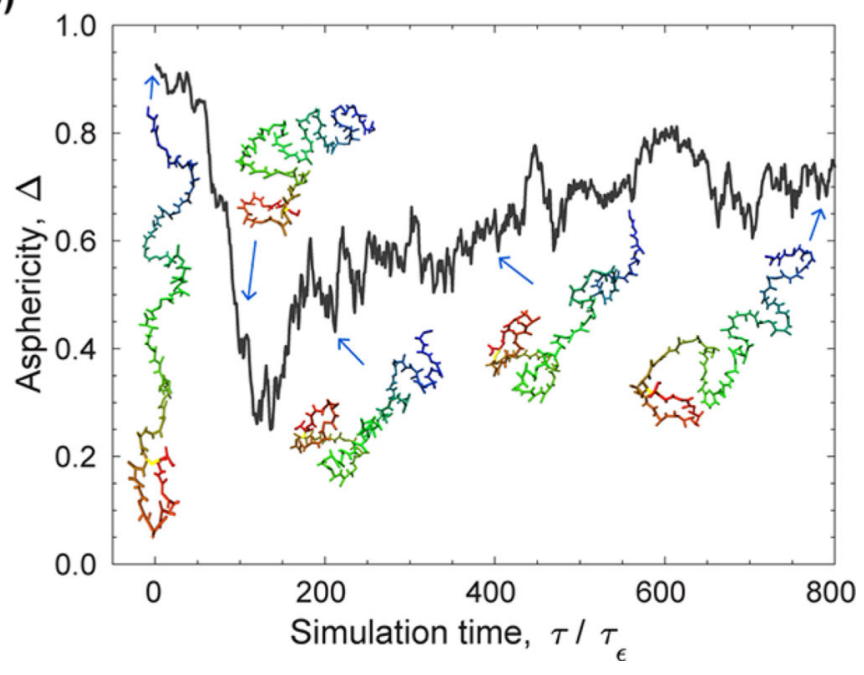

Figure 11.

Initial stage of two canonical simulations for the model DX20A-AR8 at $k_{\mathrm{B}} T_{\mathrm{u}} / \varepsilon=1.14$ : one starting from (a) a compact configuration and the other from (b) an extended configuration. The snapshots of the protein depict the temporal structural changes. 
Table 1.

Physical Characteristics of the Coarse-Grained Model of Dextran $20^{a}$

\begin{tabular}{llcc}
\hline \multicolumn{1}{c}{ name } & AR & $\begin{array}{c}\text { number of } \\
\text { beads }\end{array}$ & $\begin{array}{c}\text { radii of beads } \\
(\boldsymbol{\sigma})\end{array}$ \\
DX20-S and DX20A-S & 0 & 1 & 8.3 \\
DX20-AR1 and DX20A-AR1 & 1 & 3 & 6.1 \\
DX20-AR2 and DX20A-AR2 & 2 & 5 & 5.3 \\
DX20-AR4 and DX20A-AR4 & 4 & 9 & 4.4 \\
DX20-AR8 and DX20A-AR8 & 8 & 17 & 3.6 \\
DX20-AR16 and DX20A-AR16 & 16 & 33 & 2.9 \\
\hline
\end{tabular}

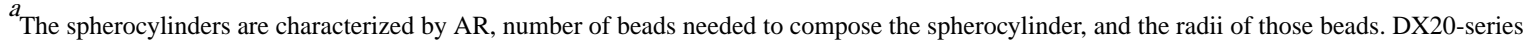
are crowders with repulsive interactions with a protein. DX20A-series are crowders with attractive interactions with a protein. 